\title{
High Exhaustion in Geriatric Healthcare Professionals During the COVID-19 Second Lockdown
}

\author{
Mohamad El Haj ${ }^{\mathrm{a}, \mathrm{b}, \mathrm{c}, *}$, Philippe Allain ${ }^{\mathrm{d}, \mathrm{e}}$, Cédric Annweiler ${ }^{\mathrm{d}, \mathrm{f}}$, Claire Boutoleau-Bretonnière ${ }^{\mathrm{g}, \mathrm{h}}$, \\ Guillaume Chapelet $^{\mathrm{i}, \mathrm{j}}$, Karim Gallouj ${ }^{\mathrm{b}}$, Dimitrios Kapogiannis ${ }^{\mathrm{k}}$, \\ Jean Roche ${ }^{1}$ and Abdel Halim Boudoukha ${ }^{a}$ \\ ${ }^{a}$ Nantes Université, Univ Angers, Laboratoire de Psychologie des Pays de la Loire, Nantes, France \\ ${ }^{\mathrm{b}}$ Unité de Gériatrie, Centre Hospitalier de Tourcoing, Tourcoing, France \\ ${ }^{\mathrm{c}}$ Institut Universitaire de France, Paris, France \\ ${ }^{\mathrm{d}}$ Laboratoire de Psychologie des Pays de la Loire, SFR Confluences, UNIV Angers, Nantes Université, \\ Maison de la recherche Germaine Tillion, Angers, France \\ ${ }^{\mathrm{e}}$ Département de Neurologie, CHU Angers, Angers, France \\ ${ }^{\mathrm{f}}$ Department of Geriatric Medicine, Angers University Hospital, Angers, France \\ ${ }^{\mathrm{g}}$ CHU Nantes, Inserm CICO4, Nantes, France \\ ${ }^{\mathrm{h}}$ CHU Nantes, Département de Neurologie, Centre Mémoire de Ressources et Recherche, Nantes, France \\ ${ }^{\mathrm{i}}$ Université de Nantes, Inserm, TENS, The Enteric Nervous System in Gut and Brain Diseases, IMAD, Nantes, \\ France \\ ${ }^{\mathrm{j}}$ CHU Nantes, Clinical Gerontology Department, Bd Jacques Monod, Nantes, France \\ ${ }^{\mathrm{k}}$ Laboratory of Clinical Investigation, National Institute on Aging, Baltimore, MD, USA \\ ${ }^{1} \mathrm{CHU}$ de Lille, Department of Geriatric Psychiatry, Lille, France
}

Accepted 2 August 2021

Pre-press 17 August 2021

\begin{abstract}
.
Background: In a previous study, we assessed burnout in geriatric healthcare workers during the first lockdown that lasted from March to May 2020 in France, in response to the COVID-19 crisis.

Objective: We carried out a follow-up study to assess burnout in the same population during the second lockdown that was implemented at the end of October 2020.

Methods: We used an online survey to assess burnout in terms of exhaustion and disengagement in a sample of 58 geriatric healthcare workers.

Results: We found higher levels of exhaustion, disengagement, and burnout among geriatric healthcare workers during the second than during the first lockdown. We also found high levels of exhaustion but moderate disengagement and burnout during the second lockdown.

Conclusion: The increased exhaustion, disengagement, and burnout during the second lockdown can be attributed to the increased workload in geriatric facilities throughout this crisis and during the second lockdown due to shortage in staff and increased number of shifts and allocated duties. The high levels of exhaustion reported among geriatric healthcare workers during the second lockdown can reflect their physical fatigue, as well as their feelings of being emotionally overextended and exhausted by their workload.
\end{abstract}

Keywords: Burnout, COVID-19, disengagement, exhaustion, healthcare professionals

\footnotetext{
*Correspondence to: Mohamad El Haj, Faculté de Psychologie, LPPL - Laboratoire de Psychologie des Pays de la Loire, Université de Nantes, Chemin de la Censive du Tertre, BP 81227 ,
}

44312 Nantes Cedex 3, France. E-mail: mohamad.elhaj@univnantes.fr. 


\section{INTRODUCTION}

The current COVID-19 pandemic has added to the already high levels of stress that healthcare professionals experience. While all health professionals have had to shoulder increased burden and workload during the COVID-19 crisis, those who work in the geriatric field have not been generally recognized as being particularly affected and hence little attention has been paid to their probable burnout during the crisis. We thus assess, in the present paper, burnout, i.e., the negative physical and emotional reactions to exposure to a stressful work environment [1], of healthcare workers in geriatric facilities during the COVID-19 crisis. More specifically, we assess burnout in a French sample of geriatric healthcare workers during the second lockdown of the COVID-19 crisis; this was based on a previous study demonstrating medium levels of burnout in this population during the first lockdown [2].

A burgeoning research has begun to assess the effects of the COVID-19 crisis on stress and burnout in healthcare workers. This research has demonstrated how the crisis has put considerable physical and emotional strain (e.g., fatigue, irritability, emotional exhaustion, sleep disorders) on healthcare workers [3-11]. Among healthcare workers, those who work in the geriatric field represent a unique group because they had to deal with both the COVID-related consequences and the vulnerability of geriatric patients. Patients in geriatric facilities typically suffer from age-related functional and cognitive decline, carry multiple and often challenging diagnoses, and receive multiple treatments for agerelated medical conditions, increasing their risk for COVID-19 infection and death. The vulnerability of geriatric patients can be expected to increase the stress level of geriatric healthcare workers who care for them and, probably, their burnout during the COVID-19 crisis. This burnout can also be expected for other reasons, including increased probability of contracting COVID-19 and, consequently, passing the disease to their loved ones, increased workload, continuously changing practice environment (e.g., virtual rather than face-to-face staff meetings, telemedicine replacing face-to-face encounters), and, in some cases, lack of adequate personal protective equipment. Another factor potentially exacerbating burnout of healthcare workers in geriatric facilities during the COVID-19 crisis is social isolation and loneliness, especially for those who decided to confine themselves to the geriatric facilities with their patients to reduce the risk of contaminating patients, as well as their own families. Taken together, caring for extremely vulnerable patients, experiencing their deaths, disturbances in the work environment, long working hours, and social isolation, are factors that may result in increased burnout among healthcare workers in geriatric facilities during the COVID-19 crisis.

We assessed burnout in healthcare workers in French geriatric facilities during the first lockdown of the COVID-19 crisis that lasted from March 17 to May 11, 2020 in a previous study. [2]. In our previous study, we used an online survey based on the Oldenburg Burnout Inventory (OLBI) [12], a scale assessing burnout in terms of exhaustion (i.e., energy depletion or the draining of emotional resources) and disengagement (i.e., withdrawal and passivity toward work). Eighty-four geriatric healthcare workers (physicians, nurses, nursing assistants) answered our survey during the lockdown. Analysis of the data demonstrated medium levels of burnout, exhaustion, and disengagement. This level of burnout was attributed by the authors to the fatigue, loss of energy, and/or feelings of being overextended and exhausted. Because our previous study [2] has dealt with the first lockdown, we investigate, in the present study, levels of burnout in the same population during the second lockdown. Below we provide an overview of the social and geriatric environment during the second lockdown of the COVID-19 crisis.

Following the first lockdown, France eased restrictions from July to September 2020. However, because COVID-19 cases have spiked sharply in October, a second nationwide lockdown was imposed on October 30, 2020, and this second lockdown was eased on November 27, allowing for more freedom of movement. Compared to the first lockdown, families were allowed to visit residents in geriatric facilities in a less restrictive manner to avoid the increased isolation and psychological distress these resident experienced during the first lockdown [13-16]. However, during the second lockdown, geriatric healthcare workers had to deal with increased challenges, such as the continuous shortage in staff and departures of colleagues following the first lockdown. Also, although a form of exhilaration and social engagement was observed among caregivers and volunteers during the first lockdown, this engagement has decreased during the second lockdown, perhaps mirroring the discouragement of the general population from the duration of pandemic. Critically, the increased workload and shortage of staff, as occurred during the 
first lockdown in geriatric facilities, has persisted to the second lockdown, decreasing the opportunity of geriatric healthcare workers to have a long break and distance themselves from the workload. The accumulated long-term workload has exacerbated the stress in geriatric healthcare workers during the second lockdown, especially the long and numerous shifts, which have reduced their physical and psychological resilience. In summary, continuous shortage in staff and continuous increased workload may be expected to result in increased burnout in geriatric healthcare workers during the second lockdown compared with the first lockdown.

We expected in this study increased levels of burnout, exhaustion, and disengagement in geriatric healthcare workers during the second lockdown compared with the first lockdown. To test our hypothesis, we carried-out a follow-up study in which we assessed burnout during the second lockdown in the same population as our previous study [2]. Because follow-up research is typically fraught with difficulties to recruit the same population, our study offers a rare opportunity to investigate and describe the continuous impact of lockdown on the mental health and wellbeing of healthcare professionals.

\section{METHODS}

\section{Participants}

Among 84 participants included in the previous study during the first lockdown [2], 58 (38 women, 20 men, $M$ age $=32.89$ years, $S D=10.75)$ responded to the invitation to participate in the present study. Among the 58 participants, eighteen were nurses, seventeen were nursing assistants, thirteen were physicians, and ten were service/environment agents (agents charged of maintaining hygiene services). All participants declared working in geriatric facilities (i.e., retirement homes, medical senior-residents, hospitals, short-term geriatric units) in France.

While one may argue that the sample size in this study may be law, we believe that the sample size is fair. Using post hoc analysis with G*Power [17], we calculated sample size for Wilcoxin tests (two-tailed) based on $95 \%$ power, an estimated probability of making Type I error of 0.05 , and a medium effect size of 0.50 [18]. Analysis suggested that 47 participants would be necessary to obtain sufficient statistical power. Thus, our sample, including 58 participants, is fair to detect significant differences between the first and second lockdowns.

\section{Procedures}

We constructed an online version, using Limesurvey, of the validated paper-and-pencil French version of OLBI. We disseminated the online survey by emails or personal invitations to participants of the previous study. The survey began on November 15, 2020, and ended on November 27, 2020 (i.e., during the second lockdown in France).

We opened the survey with a message defining the objective of the study, namely, that our study assesses the work experience of geriatric healthcare workers during the second lockdown to address the COVID19 crisis. Next, we provided an informed consent form that guaranteed anonymity and confidentiality, stated that the purpose of the study was solely scientific and explained study procedures. We also provided the contact details of the first author, in case of participants wished to receive further information about the study aims or data handling. Once participants consented to participate, they were provided with sociodemographic questions on sex, age, and profession. Then, participants were presented with the 16 items of the OLBI scale, listed in Table 1. Half of the items assessed the exhaustion dimension of burnout, whereas the other half measured the disengagement dimension of burnout. Half of the items were positively worded, whereas the other half were negatively worded. Participants had to respond to each item by using a scale ranging from one point (strongly agree) to four points (strongly disagree). When analyzing the data, we reversed scores on negatively worded items so that all high scores would refer to high levels of exhaustion and disengagement. Thus, the maximum score on each one of the exhaustion and disengagement subscales was 32 points (eight items $\mathrm{x}$ four points for each subscale) and the total score for burnout was 64 points. The survey ended with an invitation to make any additional comments, following with a message thanking the participants.

Following the French validation and scoring system of the OLBI scale, scores can be considered as indicating:

- High exhaustion for scores $>23 / 32$ points.

- Medium exhaustion for scores between 16 and $23 / 32$ points

- Low exhaustion for scores $<16 / 32$ points

- High disengagement for scores $>22 / 32$ points.

- Medium disengagement for scores between 15 and $22 / 32$ points 
Table 1

The OLBI scale

\begin{tabular}{ccccc}
\hline & Strongly & Agree & Disagree & Strongly \\
disagree
\end{tabular}

I always find new and interesting aspects of my work [R]

There are days when I feel tired before work

It happens more and more often that I talk about my work in a negative way

After work, I tend to need more time than in the past in order to relax and feel better

I can tolerate the pressure of my work well [R]

Lately, I tend to think less at work and do my job automatically

I find my work to be a positive challenge [R]

During work, I often feel emotionally drained

Over time, one can become disconnected from this type of work

After work, I have enough time for my leisure activities [R]

Sometimes I feel sickened by my work tasks

After my work, I usually feel worn out and weary

This is the only type of work I can imagine myself doing [R]

Usually, I can manage the amount of my work well [R]

I feel more and more engaged in my work [R]

When I work, I usually feel energized [R]

In statements marked $[R]$ reverse coding was used - a negative response indicates high burnout and a positive answer indicates low burnout. Disengagement items are 1, 3, 6, 7, 9, 11, 13, and 15. Exhaustion items are 2, 4, 6, 8, 10, 12, 14, and 16.

- Low disengagement for scores $<15 / 32$ points

- High burnout for scores $>44 / 64$ points.

- Medium burnout for scores between 30 and 44 164 points

- Low burnout for scores $<30 / 64$ points

Note that we used the OLBI questionnaire for several reasons. This questionnaire has been validated in several occupational spheres [19] and research has highlighted its psychometrical robustness [20-22]. We also used the OLBI questionnaire because the OLBI questionnaire is relatively short, so caregivers needed to only devote a little precious time to complete the survey during the lockdown.

To test our hypothesis, we compared scores of the 58 participants across the two measures (first lockdown versus second lockdown) using Wilcoxon signed rank tests. We further compared scores with the validated cut-offs of the OLBI scale (e.g., whether the exhaustion score is significantly higher than the "high" exhaustion cut-off as set at 32 points). Note that we used non-parametric tests because ShapiroWilk tests showed non-normal distributions of data (e.g., for data related to exhaustion in the first lockdown: $W(33)=0.98, p=0.11)$. We provided effect size $(d=0.2$ can be considered a small effect size, $d=0.5$ represents a medium effect size, and $d=0.8$ refers to a large effect size [23]). The effect size was calculated for non-parametric tests following recommendations by Rosenthal and DiMatteo [24] and Ellis [25]. For all tests, level of significance was set as $p \leq 0.05$.

\section{RESULTS}

Table 2 depicts scores on the OLBI exhaustion and disengagement subscales and the total scale.

\section{More exhaustion, disengagement, and burnout during the second lockdown}

Analysis showed higher exhaustion $(Z=4.41, p<$ 0.001 , Cohen's $d=1.42)$, disengagement $(Z=3.05$, $p=0.002$, Cohen's $d=0.87)$, and burnout $(Z=5.03$, $p<0.001$, Cohen's $d=1.75$ ) during the second lockdown than during the first lockdown.

\section{High exhaustion but moderate disengagement and burnout during the second lockdown}

Regarding scores of the first lockdown, analysis showed that the mean score for exhaustion was significantly higher than the cutoff value of $16(Z=4.98$, $p<0.001$, Cohen's $d=1.73$ ) and lower than the cutoff value of $23(Z=3.06, p=0.002$, Cohen's $d=0.87)$. Thus, this mean score indicates medium levels of exhaustion. The mean score for disengagement was significantly higher than the cutoff value of 15 $(Z=2.92, p=0.003$, Cohen's $d=0.83)$ and lower than the cutoff value of $22(Z=5.54, p<0.001$, Cohen's $d=2.12$ ). Thus, this score indicates medium levels of disengagement. The score for burnout was significantly higher than the cutoff value of $30(Z=5.53$, $p<0.001$, Cohen's $d=2.06$ ) and lower than the cutoff value of $44(Z=4.48, p<0.001$, Cohen's $d=1.45$ ), indicating medium levels of burnout. 
Table 2

Mean scores on the exhaustion and disengagement subscales of the Oldenburg Burnout Inventory scale in THE geriatric healthcare workers during the first and second lockdown

\begin{tabular}{lccc}
\hline & Exhaustion & Disengagement & Burnout \\
\hline First lockdown & Mean=20.55(5.45) & Mean=17.05 (4.56) & Mean = 37.60 (8.10) \\
& Median=20.50 & Median=16.00 & Median = \\
Second lockdown & Mean=24.57(4.23) & Mean=18.98 (5.38) & Mean =43.55 (7.03) \\
& Median=26.00 & Median=18.00 & Median= \\
$p$ values (first versus second lockdown comparison) & $p<0.001$ & $p=0.002$ & $p<0.001$ \\
\hline
\end{tabular}

Standard deviations are given between parentheses; the maximum score on each subscale was 32 points; burnout score was the total score (exhaustion + disengagement subscales) and the maximum score 64 points.

Regarding scores of the second lockdown, analysis showed that the mean score for exhaustion, as provided in Table 2, was significantly higher than the cutoff value of $23(Z=2.71, p=0.007$, Cohen's $d=0.76$ ), indicating high levels of exhaustion. The mean score for disengagement was significantly higher than the cutoff value of $15(Z=4.76, p<0.001$, Cohen's $d=1.60)$ and lower than the cutoff value of $22(Z=3.53, p<0.001$, Cohen's $d=1.05)$. Thus, this score indicates medium levels of disengagement. No significant differences were observed between the score for burnout and the cutoff value of $44(Z=0.61$, $p=0.54$, Cohen's $d=0.16$ ), indicating medium levels of burnout.

\section{Complementary analysis}

We compared burnout scores during the first lockdown between the professional categories of participants (i.e., nurses: $M=37.36, S D=(5.21)$, nursing assistants: $M=37.98, S D=(5.11)$, physicians: $M=37.11, S D=(5.18)$, service agents: $M=38.21$, $S D=(5.62))$ and found no significant differences between these categories $\left(\chi^{2}(1, N=58)=0.41\right.$, $p=0.52$ ). We also found no significant differences regarding burnout scores during the second lockdown (i.e., nurses: $M=44.35, S D=(7.98)$, nursing assistants: $M=43.96, S D=(7.62)$, physicians: $M=44.21$, $S D=(7.88)$, service agents: $M=41.71, S D=(6.74)$, $\left.\chi^{2}(1, N=58)=0.61, p=0.43\right)$.

To ensure that there are no significant differences regarding the baseline scores of the sample in the present study (i.e., the 58 participants) and scores of the sample in the previous study (i.e., the 84 participants), we compared the baseline scores between the two populations. We used Welch's $T$-test because of the unequal sample size. Analysis demonstrated no significant differences between the two samples regarding scores of exhaustion $(t(125.92)=0.41$, $p=0.68$, disengagement $t(125.92)=0.44, p=0.66$, or burnout $t(125.92)=0.53, p=0.60)$. Thus, the follow-up scores cannot be attributed to differences in baseline scores between the sample in our present study and that enrolled in our previous study [2].

\section{DISCUSSION}

Considering the possibility of increased workload during the second lockdown in the COVID-19 crisis, we assessed burnout in geriatric healthcare workers that had participated in a study assessing burnout during the first lockdown. We found that these workers experienced higher levels of exhaustion, disengagement, and burnout during the second compared to the first lockdown. Analysis also demonstrated high levels of exhaustion but moderate levels of disengagement and burnout during the second lockdown.

The higher levels of exhaustion, disengagement, and burnout during the second compared to the first lockdown, as observed in our study, may be attributed to the increased workload during the second lockdown and the accumulation of workload throughout the COVID-19 crisis. For instance, the departure of colleagues due to burnout and difficulties encountered during the first lockdown has exacerbated the workload of geriatric healthcare workers during the second lockdown. Also, and compared with the first lockdown, geriatric healthcare workers were required to perform more tasks (e.g., reinforced hygiene precautions, such as wearing full personal protective equipment and being tested before every shift, increased frequency of screening for potential fever and respiratory symptoms in residents), increasing their shifts and decreasing time that they can dedicate to their physical, psychological, and social wellbeing. Although geriatric facilities, at least in France, promised increased wages after the first lockdown, little effort has been made to improve these wages, with only a slight improvement being made in the public health sector, exacerbating the frustration in geriatric healthcare workers during the 
second lockdown. This burnout can also be attributed to the decreased motivation and discouragement of the general population during the second lockdown, probably because of the long duration of pandemic. For instance, in France, people under lockdown were showing their gratitude to front-line healthcare workers worldwide by applauding them each day at 8 PM. However, this gesture was only offered during the first lockdown.

In addition, although geriatric facilities were better prepared to implement new care and infection protocols during the second lockdown, healthcare workers have encountered some difficulties implementing these tasks due to shortage of staff and equipment and relatively low level of training before the second lockdown. Moreover, the rapid change in routines for care and modified work guidance, content, and environment, may have led to uncertainty and confusion. Exacerbating the geriatric healthcare workers' exhaustion may be the increased cognitive and behavioral problems encountered by their patients, especially those in the long-term care facilities who suffered the consequences of both the first and second lockdowns. To cope with COVID-19 and limit its spread among residents, geriatric facilities have been obliged to reduce physical contact between patients and families and, in some cases, between patients and caregivers, thereby exacerbating their feelings of loneliness. Another reason, although anecdotal, that may potentially explain the higher levels of burnout during the second lockdown was indicated by a participant as a comment after the end of the questionnaire: compared with the first lockdown that occurred between March and May 2020, the second lockdown occurred during the Autumn with shorter days and less daylight, factors that are known to trigger seasonal affective disorder or more broadly negatively affect mood.

While our participants reported moderate levels of disengagement and burnout during the first and second lockdown, they reported higher level of exhaustion during the second lockdown. This higher level of exhaustion may reflect the fatigue and loss of energy, as well as their feelings of being emotionally overextended and exhausted by the workload. These feelings are commonly being described by healthcare workers as being "worn out", "empty", or "feeling used up at the end of the shift". Emotional exhaustion is particularly problematic for healthcare professionals, as it may interfere with their ability to show empathy to their patients [26], especially the most vulnerable among them, such as elderly. Regarding the medium levels of disengagement observed during the first and second lockdowns, these levels may reflect some tendency of the participants to distance themselves from the object and the content of their work, as well as some negative attitudes toward their work. Nevertheless, the fact that levels of disengagement and burnout are no worse than medium can be, somehow, surprising and may even be considered as relatively good news, as one may have expected even higher levels of disengagement and burnout during the second lockdown. Due to the increased workload and since the number of patients' deaths during the COVID-19 crisis has increased in geriatric facilities, geriatric healthcare workers have been exposed to increased stress. Although this stress may be expected to induce burnout [27], our participants have only demonstrated medium levels of disengagement and burnout, probably mirroring their devotion to their profession, as well as some levels of resilience.

The medium levels of burnout during the second lockdown, as reported by our participants, are by no means negligible. Burnout can generate psychological disturbances with serious impact on the health and wellness, including lower self-esteem, worsening anxiety, and frustration, and, potentially, insomnia, headaches, pain, gastrointestinal problems, as well as the potential for consumption of high levels of caffeine or even tranquilizers and illicit drugs [28, 29]. Such consequences of burnout may not only impact the mental and physical health of geriatric healthcare workers, but also the quality of care they provide. We thus believe that it is important to implement strategies to decrease burnout among geriatric healthcare workers during and after the COVID-19 crisis. Even during the crisis, geriatric healthcare workers should be enabled to stay more connected with their families and friends (providing necessary technology, time, and resources if necessary). Geriatric healthcare workers should also, whenever possible, take breaks or breathers, and exercise, or perhaps use mindfulness, as research has demonstrated its positive effects on burnout [30-33]. Employers should provide geriatric healthcare workers with resources for support, including medical and psychological professional help.

In short, geriatric healthcare workers are dealing with a demanding, unprecedented, and stressful situation during the COVID-19 crisis. Their exhaustion during the second lockdown may not only negatively impact their wellbeing, but also the quality of patient care. Although the major focus during the COVID19 crisis is on addressing the spread of the virus, 
the needs of healthcare workers in general, including those in the geriatric field, also need to be taken into consideration and addressed. This is especially important in case of any other (upcoming) lockdown.

\section{ACKNOWLEDGMENTS}

The authors would like to thank the caregivers who, despite the increased workload during the COVID19 crisis, have dedicated their time to answer the survey. Dr El Haj was supported by the LABEX (excellence laboratory, program investment for the future) DISTALZ (Development of Innovative Strategies for a Transdisciplinary Approach to Alzheimer Disease), and the EU Interreg CASCADE 2 Seas Programme 2014-2020 (co-funded by the European Regional Development Fund). This research was supported in part (for author DK) by the Intramural Research Program of the National Institute on Aging, NIH.

Authors' disclosures available online (https:// www.j-alz.com/manuscript-disclosures/21-0615r1).

\section{REFERENCES}

[1] Freudenberger HJ (1974) Staff burn-out. J Soc Issues 30, 159-165.

[2] El Haj M, Allain P, Annweiler C, Boutoleau-Bretonnière C, Chapelet G, Gallouj K, Kapogiannis D, Roche J, Boudoukha AH (2020) Burnout of healthcare workers in acute care geriatric facilities during the COVID-19 crisis: An online-based study. J Alzheimers Dis 78, 847-852.

[3] Morgantini LA, Naha U, Wang H, Francavilla S, Acar O, Flores JM, Crivellaro S, Moreira D, Abern M, Eklund M, Vigneswaran HT, Weine SM (2020) Factors contributing to healthcare professional burnout during the COVID-19 pandemic: A rapid turnaround global survey. PLoS One 15, e0238217.

[4] Algunmeeyn A, El-Dahiyat F, Altakhineh MM, Azab M, Babar ZU (2020) Understanding the factors influencing healthcare providers' burnout during the outbreak of COVID-19 in Jordanian hospitals. J Pharm Policy Pract 13, 53.

[5] Bradley M, Chahar P (2020) Burnout of healthcare providers during COVID-19. Cleve Clin J Med, doi: 10.3949/ccjm. 87 a.ccc051

[6] Shanafelt T, Ripp J, Trockel M (2020) Understanding and addressing sources of anxiety among health care professionals during the COVID-19 pandemic. JAMA 323, 2133-2134.

[7] Lai J, Ma S, Wang Y, Cai Z, Hu J, Wei N, Wu J, Du H, Chen T, Li R, Tan H, Kang L, Yao L, Huang M, Wang H, Wang G, Liu Z, Hu S (2020) Factors Associated with mental health outcomes among health care workers exposed to coronavirus disease 2019. JAMA Network Open 3, e203976.

[8] Yildirim M, Solmaz F (2020) COVID-19 burnout, COVID19 stress and resilience: Initial psychometric properties of COVID-19 Burnout Scale. Death Stud, doi: 10.1080/ 07481187.2020.1818885
[9] Ruiz-Fernandez MD, Ramos-Pichardo JD, Ibanez-Masero O, Cabrera-Troya J, Carmona-Rega MI, Ortega-Galan AM (2020) Compassion fatigue, burnout, compassion satisfaction and perceived stress in healthcare professionals during the COVID-19 health crisis in Spain. J Clin Nurs 29, 43214330.

[10] Di Monte C, Monaco S, Mariani R, Di Trani M (2020) From resilience to burnout: Psychological features of Italian general practitioners during COVID-19 emergency. Front Psychol 11, 567201.

[11] Luceno-Moreno L, Talavera-Velasco B, Garcia-Albuerne Y, Martin-Garcia J (2020) Symptoms of posttraumatic stress, anxiety, depression, levels of resilience and burnout in Spanish health personnel during the COVID-19 pandemic. Int $J$ Environ Res Public Health 17, 5514.

[12] Demerouti E, Nachreiner F (1998) Zur Spezifität von burnout für dienstleistungsberufe: Fakt oder artefakt [The specificity of burnout for human services: Fact or artefact]. $Z$ Arbeitswiss 52, 82-89.

[13] Boutoleau-Bretonnière $\mathrm{C}$, Pouclet-Courtemanche H, Gillet A, Bernard A, Deruet AL, Gouraud I, Mazoue A, Lamy E, Rocher L, Kapogiannis D, El Haj M (2020) The effects of confinement on neuropsychiatric symptoms in Alzheimer's disease during the COVID-19 crisis. J Alzheimers Dis 76, 41-47.

[14] Boutoleau-Bretonnière C, Pouclet-Courtemanche H, Gillet A, Bernard A, Deruet AL, Gouraud I, Lamy E, Mazoué A, Rocher L, Bretonnière C, El Haj M (2020) Impact of confinement on the burden of caregivers of patients with the behavioral variant of frontotemporal dementia and Alzheimer disease during the COVID-19 crisis in France. Dement Geriatr Cogn Disord Extra 10, 127-134.

[15] El Haj M, Altintas E, Chapelet G, Kapogiannis D, Gallouj K (2020) High depression and anxiety in people with Alzheimer's disease living in retirement homes during the Covid-19 crisis. Psychiatry Res 291, 113294.

[16] El Haj M, Larøi F, Gallouj K (2020) Hallucinations in a patient with Alzheimer's disease during the COVID-19 crisis: A case study. J Alzheimers Dis Rep 4, 455-458.

[17] Faul F, Erdfelder E, Lang AG, Buchner A (2007) G*Power 3: A flexible statistical power analysis program for the social, behavioral, and biomedical sciences. Behav Res Methods 39, 175-191.

[18] Cohen J (1992) Statistical power analysis. Curr Dir Psychol Sci 1, 98-101.

[19] Reis D, Xanthopoulou D, Tsaousis I (2015) Measuring job and academic burnout with the Oldenburg Burnout Inventory (OLBI): Factorial invariance across samples and countries. Burnout Res 2, 8-18.

[20] Halbesleben JRB, Demerouti E (2005) The construct validity of an alternative measure of burnout: Investigating the English translation of the Oldenburg Burnout Inventory. Work Stress 19, 208-220.

[21] Halbesleben JRB (2010) The role of exhaustion and workarounds in predicting occupational injuries: A crosslagged panel study of health care professionals. J Occup Health Psychol 15, 1-16.

[22] Timms C, Brough P, Graham D (2012) Burnt-out but engaged: The co-existence of psychological burnout and engagement. $J$ Educ Admin 50, 327-345.

[23] Cohen J (1988) Statistical power analysis for the behavioral sciences, Erlbaum Associates, Hillsdale, NJ.

[24] Rosenthal R, DiMatteo MR (2001) Meta-analysis: Recent developments in quantitative methods for literature reviews. Annu Rev Psychol 52, 59-82. 
[25] Ellis PD (2010) The Essential Guide to Effect Sizes: Statistical Power, Meta-Analysis, and the Interpretation of Research Results., Cambridge University Press, New York, NY.

[26] Boudoukha AH, Denis N, Zinger-Maurin M (2020) Burnout et médecine palliative : Souffrances psychiques des internes. Burnout and palliative medicine. Médecine Palliative 20, 135-143.

[27] Boudoukha AH, Altintas E, Rusinek S, Fantini-Hauwel C, Hautekeete M (2013) Inmates-to-staff assaults, PTSD and burnout: Profiles of risk and vulnerability. J Interpers Violence 28, 2332-2350.

[28] Cherniss C (1992) Long-term consequences of burnout: An exploratory study. J Organ Behav 13, 1-11.

[29] Weber A, Jaekel-Reinhard A (2000) Burnout syndrome: A disease of modern societies? Occup Med (Lond) 50, 512-517.
[30] Cohen-Katz J, Wiley S, Capuano T, Baker DM, Deitrick L, Shapiro S (2005) The effects of mindfulness-based stress reduction on nurse stress and burnout: A qualitative and quantitative study, part III. Holist Nurs Pract 19, 78-86.

[31] Goodman MJ, Schorling JB (2012) A mindfulness course decreases burnout and improves well-being among healthcare providers. Int J Psychiatry Med 43, 119-128.

[32] Irving JA, Dobkin PL, Park J (2009) Cultivating mindfulness in health care professionals: A review of empirical studies of mindfulness-based stress reduction (MBSR). Complement Ther Clin Pract 15, 61-66.

[33] Singh NN, Lancioni GE, Karazsia BT, Chan J, Winton AS (2016) Effectiveness of caregiver training in mindfulnessbased positive behavior support (MBPBS) vs. training-asusual (TAU): A randomized controlled trial. Front Psychol 7, 1549 . 